\title{
Primary Tuberculous Osteomyelitis of the Mandible Mimicking a Parotid Gland Abscess
}

\author{
Deepak Dalmia $^{1}$ (1) $\cdot$ Pankil Shah $^{1} \cdot$ Jeena Pillai $^{1}$
}

Received: 19 August 2015/Accepted: 8 February 2016/Published online: 22 February 2016

(C) Association of Otolaryngologists of India 2016

\begin{abstract}
Tuberculosis has become a rare disease in the developed countries but it is still a serious problem in the developing countries. Incidence of tuberculous osteomyelitis of the jaw bones is very low. This rare incidence is the primary reason that the lesion gets misdiagnosed many times. Here we report the diagnosis, treatment and follow up of a case which is not a classical presentation of tuberculous osteomyelitis of mandible. Early diagnosis of tuberculous osteomyelitis will certainly reduce the morbidity of this disease condition.
\end{abstract}

\section{Keywords Mandible $\cdot$ Osteomyelitis $\cdot$ Tuberculosis}

\section{Introduction}

Tuberculous osteomyelitis of the mandible is a rare entity; however the resurgence in the incidence of tuberculosis due to coinfection with HIV has brought back the focus on this age old disease [1]. Oral tuberculosis is usually secondary to pulmonary involvement however the systemic manifestations may be absent and oral lesion may be the presenting finding.

Deepak Dalmia

pani1220660@gmail.com

Pankil Shah

rinalshah_89@yahoo.com

1 Dr Baba Saheb Ambedkar Memorial Central Railway Hospital, 37, no OPD, Ground Floor, Dr Baba Saheb Ambedkar Memorial Hospital, Byculla (E), Mumbai 400027, India

\section{Case Report}

A 21 year old female, residing at Byculla, Mumbai, presented with the complain of painless swelling over right side parotid region for 4-5 days in duration. It was recurrent swelling, since 8-9 months. Repeated aspirations were done, whose pus culture turned out to be sterile. It was not relieved by repeated course of the antibiotics. It was not associated with the fever, facial weakness, dryness of mouth. She had no past history tuberculosis or tuberculosis contact, weight loss, chronic cough, neck swelling and discharging sinuses.

Her general and systemic examination revealed normal findings.

On examination she had a swelling of $3 \times 3 \mathrm{~cm}$ over right parotid region with normal over lying skin, smooth, non mobile, not fixed to the over lying skin, non fluctuant, not translucent, no scar and no sinuses.

Patient's complete blood counts, ESR, LFT, RFT were within normal limits. Patient was negative for HIV, HCV, HBsAg. Patient got chest xray done which was normal (Fig. 1). Patient got orthopantogram done which was normal (Fig. 2). The first ultrasonography of the parotid region was done on 4/4/13 which revealedparotid gland showing a well outlined echogenic fluid containing area which may be due to parotid abscess. Repeated on 22/4/13 revealed $3.21 \times 1.61 \times 3.01 \mathrm{~cm}$ size fluid containing area. Aspiration was done and pus was sent for culture and sensitivity which came to be sterile. Again the swelling recurred, whose ultrasonography on 25/4/13 revealed similar findings as that of 22/4/ 13. Pus was aspirated again and was sent for cytology, AFB stain-mainly pus cells, polymorphs, lymphocytes and plasma cells, predominant were lymphocytes. AFB was negative. 


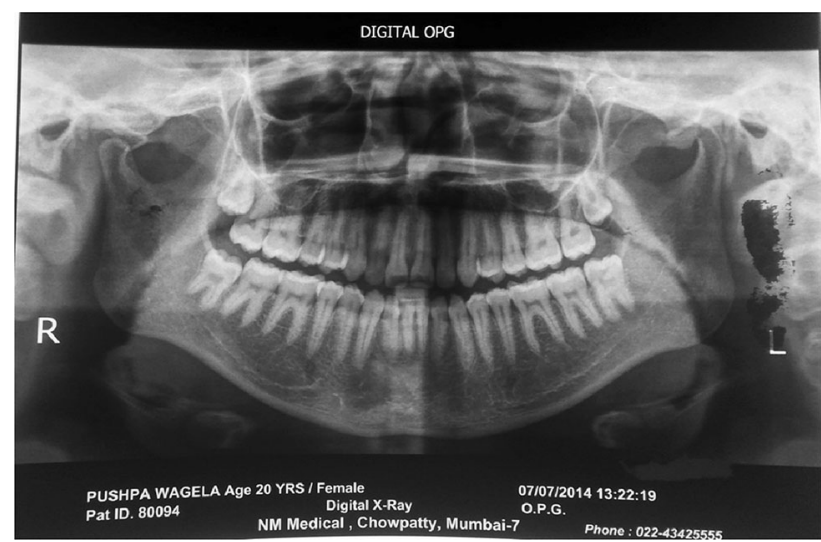

Fig. 1 Digital OPG which was normal

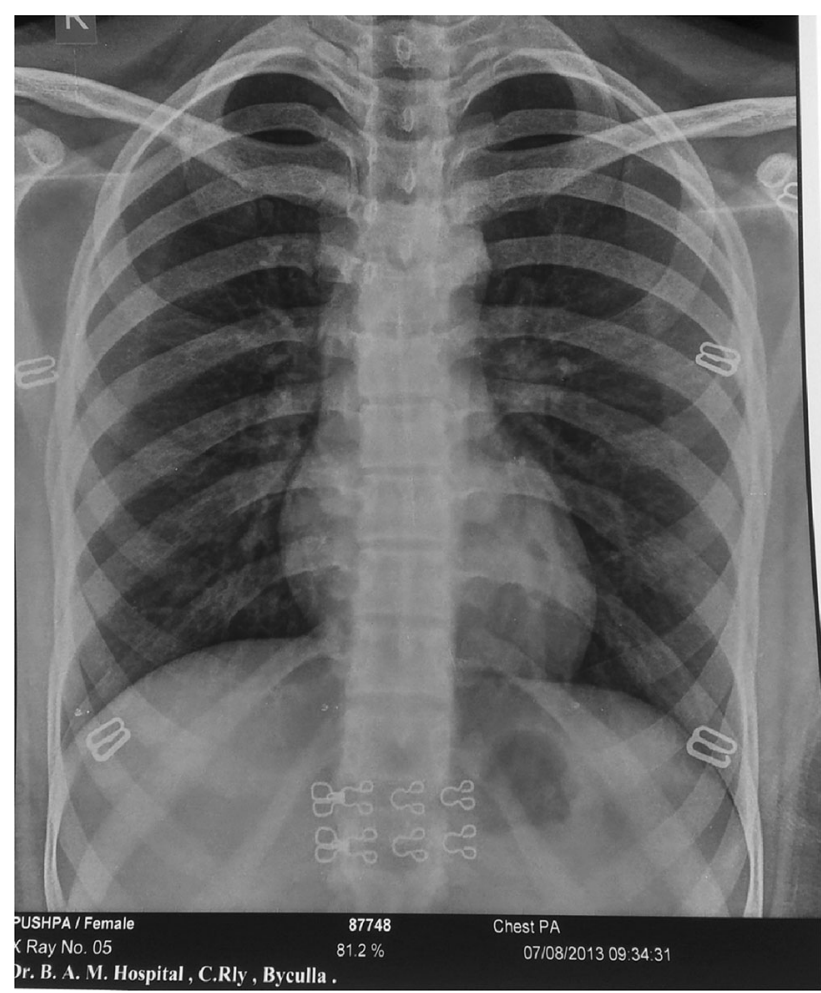

Fig. 2 Chest X-ray which was normal
Osteomyelitis of right hemimandible with masseteric abscess. Coronal STIR MRI shows altered signal intensity in the vertical ramus of right hemimandible (arrow in Fig. 3a) with an associated well defined water intensity (bright) collection (arrow in Fig. 3b) in the adjacent mas abscess.

Post-gadolinium axial MRI shows enhancing walls of the collection typical of an abscess (Fig. 3c). Two subcentimeter sized intraparotid lymphnode noted in the right parotid gland. Imaging features suggestive of an infective etiology.

Following which pus was sent for TB-PCR which detected mycobacterium tuberculosis complex.

Following which the patient was started on category 1 Anti tuberculosis treatment and responded well.

Post AKT MRI was done on 23/9/14 which showed no obvious residual lesion involving the ramus of the mandible or associated soft tissue component involving the masseter muscle on the right side at present scan (Fig. 4).

\section{Discussion}

Tuberculous osteomyelitis of the mandible is a rare entity. However in a country like India where tuberculosis is endemic, it should be an important differential when considering jaw swellings of long duration.

The disease most commonly involves young children. Mandible is rarely involved due to the less amount of cancellous bone [2]. The chief routes of spread are from infected sputum, surrounding soft tissue and haematogenous route. Wound following extraction of a tooth is also a possible conduit. The angle and the ramus of mandible are commonly involved. Destruction and erosion of the cortex occurs which is replaced by soft granulation tissue. Caseation occurs resulting in softening, liquefaction and formation of a subperiosteal abscess which can either burst intraorally or extraorally [3]. Blurring of the bony details is the first sign and later a radiolucent area can also be seen 


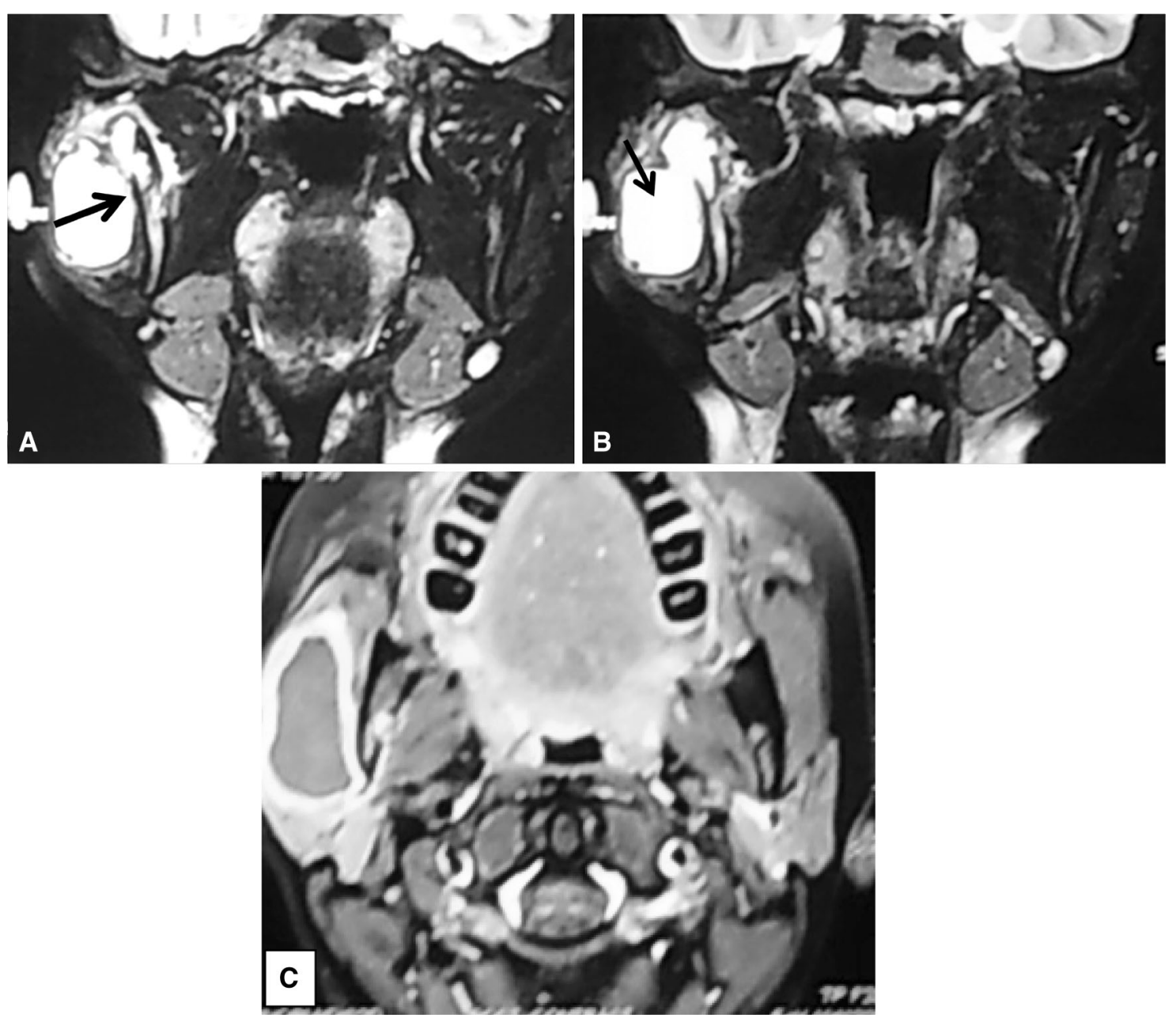

Fig. 3 Pre AKT MRI

on CT due to decalcification. The diagnosis is difficult in children because in its primary form it is paucibacillary and bacteriological correlation is always lacking. Considering the overall prevalence of TB in India, the presence of epithelioid cell granuloma is indicative of TB unless proven otherwise [4].
The treatment if initiated early results in complete resolution of the lesion. According to RNTCP the treatment advocated is of 6 months comprising of an intensive therapy of 2 months with Isoniazid, Rifampicin and Pyrazinamide and a maintenance therapy of 4 months with Rifampicin and Isoniazid. 

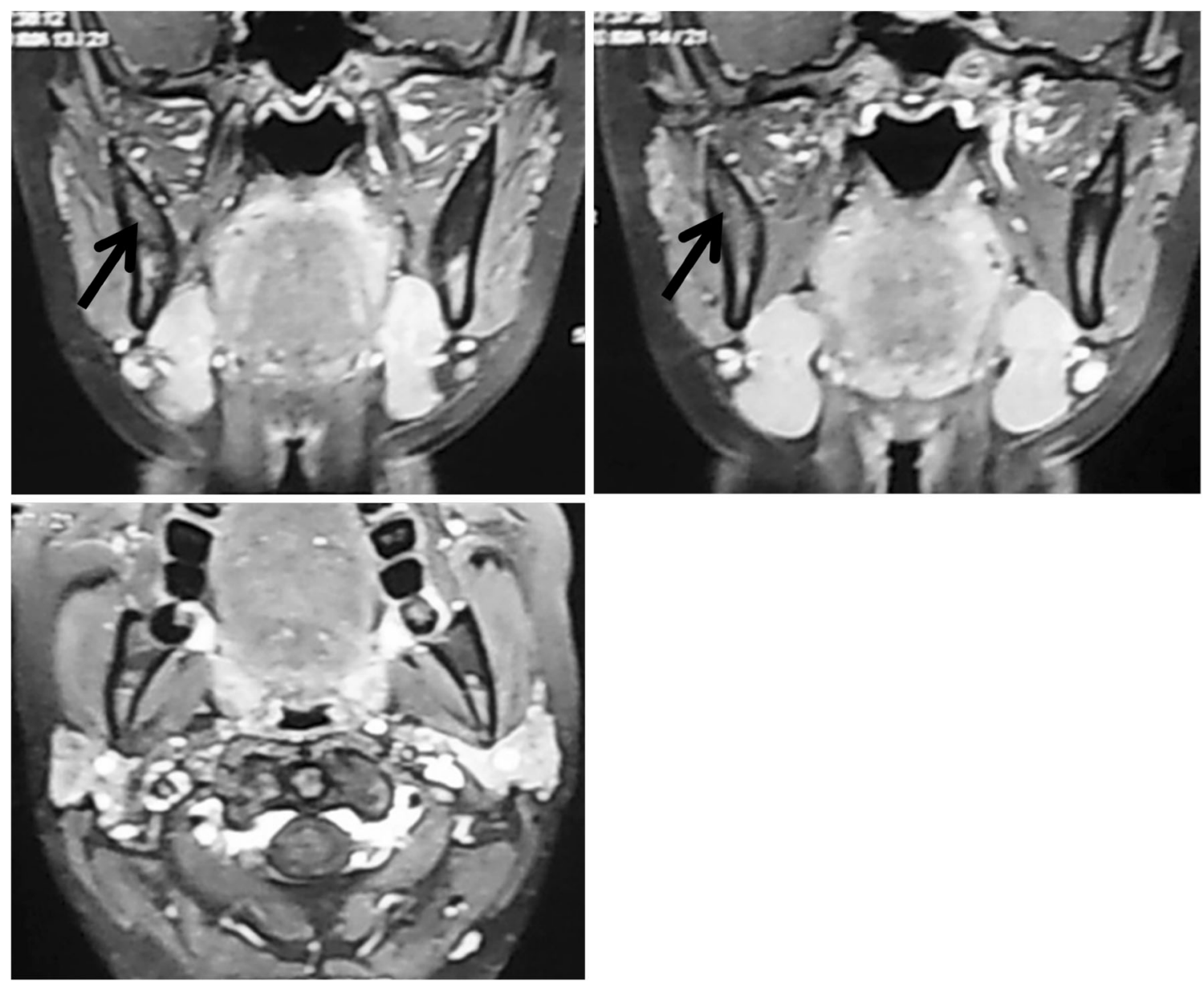

Fig. 4 Post AKT Resolution of the lessions

\section{Compliance with Ethical Standards}

Conflict of interest The authors declare that they have no conflict of interest.

Humans and Animals Rights All applicable international, national, and/or institutional guidelines for the care and use of the human participant were followed.

Informed Consent Informed consent was obtained from the participant included in the study.

\section{References}

1. Chaudhary S, Kalra N, Gomber S (2004) Tuberculous osteomyelitis of the mandible: case report in a 4 year old child. Oral Surg Oral Med Oral Pathol Oral Radiol Endodontol 97(5):603-606

2. Sachs SA, Eisenbud L (1977) Tuberculous osteomyelitis of the mandible. Oral Surg 44:425-426

3. Bhatt AP, Jaykrishnan A (2001) Tuberculous osteomyelitis of the mandible: a case report. Int J Paediatr Dent 11(4):304-308

4. Pandit AA, Khilani PH, Prayag AS (1995) Tuberculous lymphadenitis, extended cytomorphological features. Diagn Cytopathol 12:23 